\title{
Expression of hunchback during oogenesis and embryogenesis in Locusta migratoria manilensis (Meyen)
}

\author{
HE ZhengBo ${ }^{1 *}$, CAO YueQing ${ }^{2}$, CHEN Bin ${ }^{1} \&$ LI TingJing ${ }^{1}$ \\ ${ }^{1}$ Institute of Insect \& Molecular Biology, Chongqing Normal University, Chongqing 400047, China; \\ ${ }^{2}$ College of Bioengineering, Chongqing University, Chongqing 400030, China
}

Received January 8, 2010; accepted January 11, 2010

\begin{abstract}
$h b$ (hunchback) is a contributing factor in anteroposterior axial patterning of insects. Although the hb function in Locusta migratoria manilensis has been investigated, its expression pattern remains unknown. Here, the mouse polyclonal antibody was produced against $\mathrm{Hb}$ fusion protein, and then its expression pattern during oogenesis and embryogenesis of L. migratoria manilensis was examined by immunohistochemical staining. Hb protein was detected in the oocyte nucleus which was positioned centrally within the developing oocyte. The oocyte nucleus gradually moved to the posterior end of the egg along with the oocyte maturing. In freshly laid eggs, Hb formed gradient at the posterior end of the egg, and then $h b$ was expressed as a band in the middle of the blastodisc. As the blastodisc differentiated into the head and trunk, the expression region became wide, which would develop into spatial gnathal and thoracic segments. With abdominal segmentation, the expression domain in the gnathal and thoracic region became faint and eventually faded out, while the Hb expression domain appeared at the posterior growth zone in a discontinuous expression manner. The $h b$ expression pattern of L. migratoria manilensis is greatly similar to that of other locusts, such as Schistocerca americana and another L. migratoria. Compared with other insects, hb expression is conserved in the gnathal and thoracic domains, while divergent in oogenesis and abdomen.
\end{abstract}

Locusta migratoria manilensis (Meyen), hunchback, antibody production, embryogenesis, expression pattern

Citation: $\quad$ He Z B, Cao Y Q, Chen B, et al. Expression of hunchback during oogenesis and embryogenesis in Locusta migratoria manilensis (Meyen). Sci China Life Sci, 2011, 54: 146-151, doi: 10.1007/s11427-010-4128-8

Though all insects possess a highly conserved adult body plan, insects vary significantly in how the segmented embryo is formed [1,2]. Three modes of embryogenesis are generally recognized: short, intermediate, and long germ embryogenesis. In long germ embryos like Drosophila melanogaster and Nasonia vitripennis, all segments are formed simultaneously during the blastoderm stage within the early syncytial environment of the embryo. By contrast, in short and intermediate germ embryos (both described as 'short' hereafter), for example, Tribolium castaneum, Gryllus bimaculatus and Oncopeltus fasciatus, only the anterior segments are formed in the blastoderm stage, while poste-

*Corresponding author (email: zhengbohe@ yahoo.com.cn) rior segments arise during the subsequent development. Long germ band modes are restricted to the higher insects, whereas the short germ embryos are widely represented among insects, indicating that short germ band development is ancestral $[2,3]$.

The molecular mechanisms of patterning and segmentation during Drosophila embryogenesis have been studied a lot, in which the segmentation depends on progressive actions of maternal coordinate, gap, pair-rule and segment polarity genes $[1,4,5]$. The striking differences between short and long germ band embryology imply fundamental differences in patterning at the molecular level [6]. For example, the grasshopper even-skipped orthologue is not expressed in a pair-rule pattern, raising the possibility that the 
upstream regulatory genes of even-skipped may play different roles in short and long germ band insects [7].

$h b$ (hunchback) functions as both maternal gene and gap gene, which encodes a zinc-finger-containing transcription factor known to be critical for controlling other gap genes as well as pair-rule and homeotic genes [8-13]. The Drosophila embryo mutant for zygotic $h b$ exhibits a canonical gap defect, i.e., deletion of the labial through the metathoracic segments, and fusion of the A7/A8 abdominal segments [14]. Knockdown of $h b$ in coleopteran $T$. castaneum by parental RNAi results in deletion of the gnathal and thoracic segments [15]. N. vitripennis $h b$ mutant, headless, deletes all of the head except the most anterior labral segment, as well as thoracic and posterior A7-A10 segments [16,17]. By contrast, $h b$ RNAi in $O$. fasciatus does not delete the gnathal and thoracic segments but transforms them toward abdominal identity, at the same time also severely disrupts abdominal growth and segmentation [2]. Similarly, the gnathal and thoracic regions are transformed towards the abdominal identity, and only three abdominal segments are formed in severe cases of $G$. bimaculatus $h b$ RNAi depletion [18]. $h b$ functions as canonical gap gene in Drosophila, Tribolium and Nasonia, while exhibits non-canonical gap gene in $\mathrm{On}$ copeltus and Gryllus. These facts suggest that the $h b$ function has diversities in different insects.

Comparative studies of $h b$ expression and function in more primitive insects would be likely to elucidate the evolution of segmentation mechanisms. The migratory locust, Locusta migratoria, is an orthopteran pest and a representative member of the most basal hemimetabolous insects. Some species of the grasshopper genus Locusta are receiving increasing attention as a powerful model system in neurosciences, development and evolution [19]. We have isolated the homologue of the Drosophila gap gene $h b$ of $L$. migratoria manilensis (Lmm'hb), and investigated its function by paternal RNA interference (RNAi). Phenotypes resulting from $h b$ knockdown were categorized into three classes based on severity. In the most extreme case, embryos only developed the most anterior structures, including the labrum, antennae and eyes [6]. The expression patterns of Lmm'hb during the oogenesis and embryogenesis are still unknown. Here, we prepared the mouse polyclonal antibody against $\mathrm{Hb}$ fusion protein, and then examined the $\mathrm{Hb}$ expression pattern during oogenesis and embryogenesis at the protein level.

\section{Materials and methods}

\subsection{Experimental animals}

The colony of L. migratoria manilensis was maintained artificially with a 12-h light/12-h dark photoperiod. BALB/C mice were bought from the Laboratory Animal Center of the Third Military Medical University, China.

\subsection{Main reagents}

The pMD-hb plasmid containing full-length $L m m$ ' $h b$ cDNA sequence, pET30a(+) used to $\mathrm{Hb}$ prokaryotic expression, $E$. coli BL21 and JM109 were stored in our lab. BugBuster Protein Extraction Reagent and Ni-NTA His Bind Kit were the products of Merck (Darmstadt, Germany). Alkaline phosphatase (AP) or horseradish peroxidase (HRP)-conjugated goat antimouse IgG was purchased from Sigma (St. Louis, MO, USA). Engrailed monoclonal antibody 4D9 was purchased from Developmental Studies Hybridoma Bank (Iowa City, IA, USA). AP or HRP substrate coloration kit was the product of Dingguo Ltd. (Beijing, China). All other reagents used were of analytical grade.

\section{3 $h b$ prokaryotic expression and purification}

A $6 \times \mathrm{His} /$ L. migratoria $\mathrm{Hb}$ fusion protein was constructed by cloning a fragment, beginning at the Xho I site within the M-1 finger and continuing through to the end of the coding region, into a pET30a(+) vector. The construction process was carried out as follows: $6 \times$ His and Hind III site were added to the $3^{\prime}$ end of the $h b$ coding region by PCR. Both the PCR products and pET30a(+) were digested with Xho I and Hind III, and then the digested products were recovered and ligated at $16^{\circ} \mathrm{C}$ overnight. The ligated vector (pET30a$\mathrm{Hb}$ ) was transformed into E. coli JM109. The positive clones were identified by sequencing and digesting with the same enzymes as that used in pET30a-Hb construction. The plasmids pET30a-Hb with the correct sequence and insert direction were extracted, and then transformed into BL21 cells. After optimizing the induction conditions including IPTG, time and temperature, the fusion protein was purified according to Ni-NTA His Bind Kit's protocol.

\subsection{Hb polyclonal antibody production}

The fusion protein mixed with the same volume of freund complete adjuvant was boosted into subcutaneous sites of BALB/C mice. After the first immunization, reinforced immunization was done every two weeks. On the 7 th day after the last immunization, blood was taken from eyes. The antiserum was stored at $-70^{\circ} \mathrm{C}$.

\subsection{Western blot}

The Western blot analyses of $\mathrm{Hb}$ fusion protein and the $\mathrm{Hb}$ polyclonal antibody were carried out according to the method described previously [20].

\subsection{Embryo preparation}

Fertilized eggs were laid in damp sand and incubated at $30^{\circ} \mathrm{C}$. At this temperature, L. migratoria manilensis embryos take approximately 13 days to hatch. Embryos were 
examined at the indicated times after egg lay (AEL). The embryo preparation protocol used here was largely based on the methods of Patel [21].

\subsection{Immunohistochemistry}

Locusts eggs were collected, dechorionated, and fixed according to the established protocols as described above. The embryos were rehydrated stepwise into PBS/ $0.1 \%$ Triton $\mathrm{X}-100$ (PBT), and blocked in PBT/1\% BSA for $2 \mathrm{~h}$ at room temperature. For single antibody staining experiments, the $\mathrm{Hb}$ polyclonal antibody was used at a dilution of 1:100, and carried out at $4^{\circ} \mathrm{C}$ overnight. After washing in PBT, the alkaline phosphatase-conjugated secondary antibody at a 1:2000 dilution was added and incubated at $4^{\circ} \mathrm{C}$ overnight. After further washing, embryos were stained in BCIP/NBT solutions for 5-15 min. Following the washing in PBS, embryos were placed in $70 \%$ glycerol and viewed as wholemounts.

For the double antibody staining experiments, a BCIP/ NBT solution was washed off in several changes in PBT and blocked in PBT/1\% BSA for $2 \mathrm{~h}$ at room temperature. An En-specific cross-reactive mouse monoclonal antibody Mab4D9 (Developmental Studies Hybridoma Bank, Iowa, IA, USA) was added at a 1:50 dilution and incubated at $4^{\circ} \mathrm{C}$ overnight. After washing, the horseradish peroxidase (HRP)-conjugated secondary antibody at a 1:2000 dilution was added and incubated at $4^{\circ} \mathrm{C}$ overnight. The HRP color reaction was developed with the nickel-enhanced diaminobenzidine substrate.

\subsection{Fixation and immunohistochemistry of ovary}

The ovary of locusts was dissected in PBS and fixed for 20 min in PEM-FA fixative at room temperature. The staining method was the same as that of embryos.

\subsection{Imaging}

Wholemount embryos were viewed under a Nikon microscope (SMZ1500). Images were digitized with a CCD chip video camera using Nikon Nis elements BR 3.0 software.

\section{Results}

\section{1 $h b$ prokaryotic expression and confirmation}

$\mathrm{His} / \mathrm{Hb}$ fusion protein was expressed in E. coli and purified selectively with nickel chelate resins in step elution conditions. However, there were still some non-target proteins in the purified samples. We further carried out PAGE electrophoresis and recovered the target proteins from the gel (Figure 1). The purified proteins were transferred to the nylon-membrane for Western blotting analysis with the His monoclonal antibody. There was a single strip the same to the purified protein in size (Figure 1). Results showed that the purified proteins were the $\mathrm{Hb}$ fusion proteins that could be used as antigens to produce the polyclonal antibody against $\mathrm{Hb}$.

\subsection{Hb polyclonal antibody production and specificity confirmation}

The antiserum was produced by the immunizing mouse with $\mathrm{His} / \mathrm{Hb}$ fusion proteins. Eggs freshly oviposited and recombinant $E$. coli bacteria were lysed separately, then Western blotting was conducted to determine the specificity of the antiserum at a 1:100 dilution. A specific strip was detected from the two lysed samples (Figure 2). These results showed that the antiserum was specific to the $\mathrm{Hb}$ protein and there were not cross reactions with other antigens.

\section{3 $h b$ expression during oogenesis}

Immunostaining revealed that $h b$ was expressed within developing oocytes and localized to the oocyte nucleus which was positioned centrally within the developing oocyte (Figure $3 \mathrm{~A})$. The oocyte nucleus gradually moved to the poste-

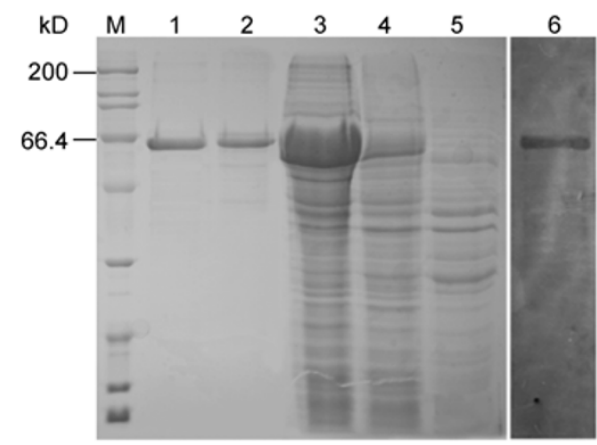

Figure 1 PAGE electrophoresis and Western blotting analysis of $\mathrm{Hb}$ fusion protein. Lane $\mathrm{M}$, protein marker; Lane $1, \mathrm{Hb}$ fusion protein recycled from PAGE gel; Lane 2, Hb fusion protein purified by affinity chromatography; Lane 3, lysates of recombinant strains after optimizing the expression conditions; Lane 4, lysates of recombinant strains before optimizing the expression conditions; Lane 5, lysates of strains with the empty vector control; Lane 6, Western blotting analysis of $\mathrm{Hb}$ fusion protein.

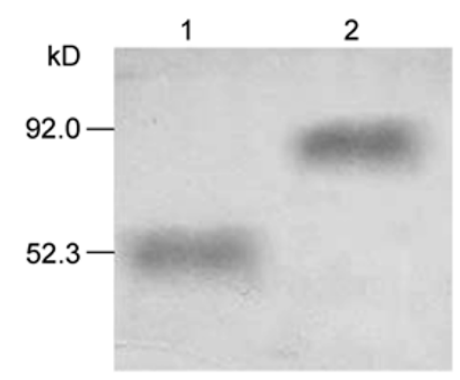

Figure 2 Analysis of antiserum specificity by Western blotting. Lane 1, lysates of recombinant strains; Lane 2, lysates of eggs freshly laid. 
rior end of the egg along with the oocyte maturing. As the nucleus moved toward the posterior end of the oocyte, $\mathrm{Hb}$ protein was still nuclear (Figure 3B and C).

\section{$2.4 h b$ expression during embryogenesis}

In freshly laid eggs, $\mathrm{Hb}$ accumulated and formed gradient at the posterior end of the egg (Figure 4A). The nuclei divided without cellular divisions at about $2 \mathrm{~h}$ AEL, migrated to the egg periphery during the next few hours, and reached the surface of the egg about $23 \mathrm{~h}$ AEL, then rapidly cellularized [22]. Around $28 \mathrm{~h} \mathrm{AEL}$, the nuclei were condensing and $h b$ expression appeared more strongly at the posterior end of the egg where the blastodisc formed (Figure 4B). Around 32 $\mathrm{h}$ AEL, the cells at the posterior end formed a circular blastodisc, and $h b$ was expressed as a band in the middle of the blastodisc (Figure 4C).

By the 42nd h AEL, the embryo differentiated into the head lobe and posterior region. At this stage, $h b$ was still expressed in the central domain, whereas absent in the head lobe and at the posterior end of the embryo (Figure 4D). With the elongations of the germband, $h b$ expression became stronger (Figure 4E and G). Till the 50th h AEL, the expression region became wide at a distinct stepped expression level (Figure 4H). By double staining with the En and $\mathrm{Hb}$ antibody, the strong region of $h b$ expression will develop into gnathal region spanning from the prospective mandibular to labial segments, while the boundary of the faint expression region will develop into the first thoracic segment (Figure 4I).

With segmentation of abdomen, the expression domain in the gnathal and thoracic region became faint and gradually faded out. $72 \mathrm{~h}$ AEL, an $\mathrm{Hb}$ expression domain appeared near the posterior end of the abdomen (Figure 4J). During the next few hours, the domain faded as the posterior region elongated, and a new domain was appearing in the more posterior region corresponding to A7-A9 abdominal segments (Figure $4 \mathrm{~K}$ ). Then another $h b$ domain appeared at the posterior of A9, which would form the 10th abdominal segment (Figure 4L and $\mathrm{M}$ ).

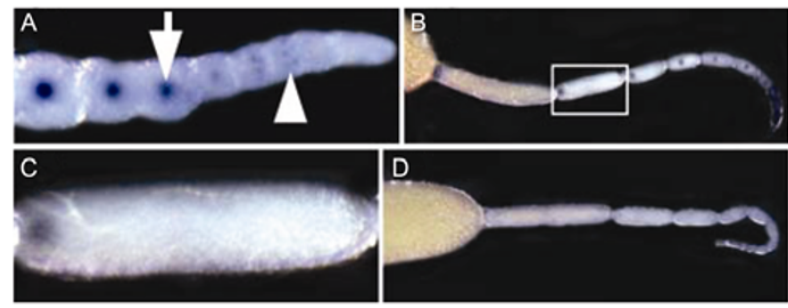

Figure 3 Maternal $h b$ expression in L. migratoria manilensis. A, Hb was expressed at the position of germarium (arrowhead), and localized to the oocyte nucleus which was positioned centrally (arrow). B, The oocyte nucleus gradually moved to the posterior end of the egg with the oocyte maturing. C, High-magnification view of the boxed area in B, showing the nucleus positioned at the posterior end of the egg. D, Ovariole staining without the primary antibody.

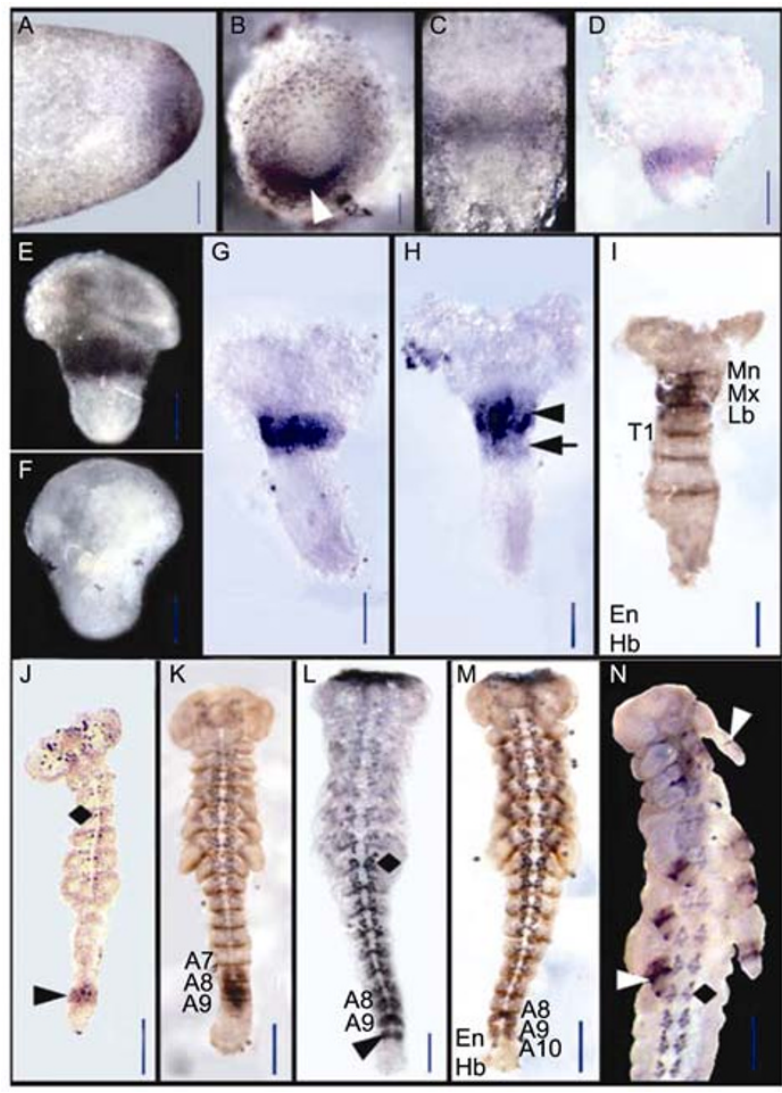

Figure $4 h b$ expression during embryogenesis. Expression patterns of $h b$ at $0(\mathrm{~A}), 28(\mathrm{~B}), 32(\mathrm{C}), 42(\mathrm{D}), 44(\mathrm{E}$ and $\mathrm{F}), 46(\mathrm{G}), 50(\mathrm{H}$ and $\mathrm{I}), 72(\mathrm{~J})$, $90(\mathrm{~K}), 96(\mathrm{~L}$ and $\mathrm{M})$ and $144 \mathrm{~h}(\mathrm{~N})$ AEL. The embryo was double-stained with En in I, $\mathrm{K}$ and $\mathrm{M}(\mathrm{Hb}$, blue; En, brown). Anterior is upwards in $\mathrm{C}-\mathrm{N}$ and towards the left in A, the frontal view of the posterior end of embryo is shown in B. A, $h b$ accumulated and formed gradient at the posterior end in freshly laid eggs. B, Blastodisc formed (arrowhead). $\mathrm{C}-\mathrm{G}, h b$ was expressed in the middle gap domain, and then gradually became wider at a distinct stepped expression level (the arrowhead indicates the stronger domain, and the arrow indicates the weak domain) (H). I, Double staining for $\mathrm{En}$ and $\mathrm{Hb}$ indicated that the strong region will develop into the gnathal region spanning from the prospective mandibular to labial segments, while the boundary of the faint expression region will develop into the first thoracic segment. The posterior expression domain (arrowheads in J) appeared at $72 \mathrm{~h} \mathrm{AEL} \mathrm{(J)} \mathrm{and} \mathrm{split} \mathrm{into} \mathrm{three} \mathrm{strips} \mathrm{corresponding} \mathrm{to} \mathrm{A7-A9} \mathrm{ab-}$ dominal segments $(\mathrm{K})$. Subsequently, the 10th abdominal segment appeared ( $\mathrm{L}$ and $\mathrm{M})$. $h b$ was also expressed on appendages indicated by arrowheads $(\mathrm{N})$, and expressed as spots or arrowheads in the head region and in the trunk indicated by diamonds $(\mathrm{J}-\mathrm{N})$. Mn, mandible; Mx, maxilla; Lb, labium; T, thoracic segments; A7-A10, abdominal segments; En, Engrailed; $\mathrm{Hb}$, Hunchback. Scale bar $=100 \mu \mathrm{m}$ in C; scale bar $=200 \mu \mathrm{m}$ in A, B, D, E, F, $\mathrm{G}, \mathrm{L}$ and $\mathrm{N}$; scale bar= $250 \mu \mathrm{m}$ in $\mathrm{H}, \mathrm{I}, \mathrm{J}, \mathrm{K}$ and $\mathrm{M}$.

When appendage primordium developed in the gnathal and thoracic segments, $h b$ was expressed strongly on the segments of the appendages, which suggested that $h b$ was probably required for the development of the appendages (Figure $4 \mathrm{~N}$ ). In the later stages, $\mathrm{Hb}$ was also expressed as spots or arrowheads in the head region and in the trunk (Figure 4J, L, and N). The expression patterns were consistent with the $\mathrm{Hb}$ neural expression in other insects [7,18,23]. These expressions suggested that $\mathrm{Hb}$ was also involved in 
neural patterning in oriental migratory locust.

\section{Discussion}

$h b$ is directly implicated in anteroposterior axial patterning of insects. Although the function of $L m m$ ' $h b$ has been investigated, its expression pattern remains unknown. The development and accumulation pattern of Lmm'hb reported here shared great similarity with that of other insects, such as Schistocerca americana and another L. migratoria [7]. Moreover, $h b$ function has been intensively investigated in several insects at different phylogenetic positions. Comparative analysis of the expression pattern and function of $h b$ in different insects may shed light on the evolutionary transition from short to long germ segmentation.

\subsection{Maternal $h b$ expressed as protein}

$h b$ functions as both maternal gene and zygotic gene. $\mathrm{Hb}$ protein was shown to be provided maternally in L. migratoria manilensis (Figure 3), consistent with that of $S$. americana [7]. However, maternal loading of $\mathrm{Hb}$ protein has not been reported in either Drosophila or Tribolium, even not in Gryllus which belongs to Orthoptera along with L. migratoria [2,15-17]. Thus the expressions of maternal $h b$ have diversities in different insects and have no consistency with phylogenetic relationships or the germband mode of insects.

In contrast to the locust, $h b$ transcript, provided by the nurse cells through cytoplasmic bridges, is not translated during oogenesis in Drosophila. Locusts have the primitive panoistic type of ovarioles, where the developing oocytes are not associated with special nurse cells. This means that $h b$ mRNA in locust oocytes is likely to be transcribed by the oocyte nucleus. In very early embryos of L. migratoria manilensis, $\mathrm{Hb}$ is only distributed in the posterior of the egg (Figure 4A), just as $S$. americana, however, $h b \mathrm{mRNA}$ is undetectable by in situ hybridization, indicating that $\mathrm{Hb}$ protein is presumably maternally provided in this developing stage [7].

In locusts, maternal $\mathrm{Hb}$ protein may only serve to distinguish embryonic cells from extraembryonic cells [7]. This contrasts with Drosophila where both maternal and zygotic $h b$ participate in axis formation, as the embryos lacking all maternal and zygotic products have a larger anterior gap than those lacking only zygotic $h b$ [14]. We also found that for $h b$, no qualitative difference in the phenotypes was produced by embryonic and parental RNAi experiments (data not shown), supporting that maternal $\mathrm{Hb}$ may not control the additional anteroposterior patterning function in locusts.

\section{2 $h b$ expression and function in the gnathal and tho- racic region}

As the germband elongated, the expression region became wider at a distinct stepped expression levels (Figure 4H), which would develop into spatial gnathal and thoracic segments. This gradient expression pattern is consistent with that of Drosophila, Gryllus, and Tribolium, indicating that $h b$ expressions in the gnathal and thoracic regions are conserved in different insects.

$h b$ functions as a gap gene in the gnathal and thoracic development in many insect species. In Drosophila, zygotic $h b$ mutants exhibit a canonical gap phenotype [14,24]. As in fruit flies, $h b$ RNAi in Tribolium results in gnathal and thoracic gap phenotypes [15]. Surprisingly, Lmm'hb RNAi results in deletions of all the gnathal and thoracic segments in class II embryos, and only the pregnathal segments are present in the extreme phenotypes [6]. These results indicate that Lmm'hb is required in more segments and might therefore function as a gap gene in a broader region than in Drosophila.

In Oncopeltus and Gryllus, the loss of gnathal and thoracic segments is thought to be due to transformation of these segments towards abdominal fates [2,18]. Interestingly, $h b$ parental RNAi in L. migratoria manilensis, an insect belonging to the same order as Gryllus, did not suppress abdominal identity, but yielded only canonical gap phenotypes. Further investigation into the expression patterns of genes involved in abdominal fates in L. migratoria will help us understand the details of the $h b$ function in $L o$ custa.

\section{3 $h b$ expression and function in abdomen}

With abdominal segmentation, the expression domain in the gnathal and thoracic region became faint and eventually faded out, and then appeared at the posterior growth zone in a discontinuous expression manner (Figure $4 \mathrm{~J}$ and $\mathrm{K}$ ). In strongly affected Lmm'hb RNAi animals, abdomen is severely compacted and segmentation is defective [6]. The $L m m$ ' $h b$ function in the developing abdominal segmentation probably reflects its expression in the posterior growth zone. Therefore, we proposed that $h b$ was required for the proper growth and segmentation of the posterior germband.

$L m m$ ' $h b$ is not continuously expressed in the growth zone, and disruption of $h b$ mRNA results in a compacted abdomen with deletions of three to four abdominal segments [6]. In Gryllus, $h b$ is expressed in the growth zone as in the locust, and required in the formation of at least seven abdominal segments [18]. By contrast, $h b$ is continuously expressed in the growth zone of two other short germband insects, Tribolium and Oncopeltus, with the compacted abdomen phenotype exhibited only in Oncopeltus. It has not yet been reported that $h b$ RNAi leads to posterior compaction in Tribolium [2]. These facts suggest that $h b$ functions in the growth zone are divergent among these species.

In short germband insects, only the anterior body segments are specified early in embryogenesis. The rest parts of the body extend later by the sequential addition of new 
segments from the growth zone, known as germ anlagen. However, the growth zone has not yet been well characterized [2]. Except for $h b$ expressed in the growth zone, other genes, such as caudal, are simultaneously expressed in this region. The functions and relationships of these genes are the key factors of growth and segmentation of posterior germband. Further analyses of the functions and relationships of these genes would be needed to elucidate the mechanisms of abdominal segmentation.

This work was supported by the National Natural Science Foundation of China (Grant No. 30700435) and Program of Natural Science Foundation of Chongqing (Grant No. CSTC2009BB1387).

1 Davis G K, Patel N H. Short, long, and beyond: molecular and embryological approaches to insect segmentation. Annu Rev Entomol, 2002, 47: 669-699

2 Liu P Z, Kaufman T C. hunchback is required for suppression of abdominal identity, and for proper germband growth and segmentation in the intermediate germband insect Oncopeltus fasciatus. Development, 2004, 131: 1515-1527

3 Sander K, Gutzeit H, Jäckle H. Insect embryogenesis: morphology, physiology, and genetical and molecular aspects. In: Kerkut G A, Gilbert L I, eds. Comprehensive Insect Physiology Biochemistry and Pharmacology, Vol. 1. Oxford: Pergamon Press, 1985. 319-385

4 Rivera-Pomar R, Jackle H. From gradients to stripes in Drosophila embryogenesis: filling in the gaps. Trends Genet, 1996, 12: 478483

5 St Johnston D, Nüsslein-Volhard C. The origin of pattern and polarity in the Drosophila embryo. Cell, 1992, 68: 201-219

$6 \mathrm{He}$ Z B, Cao Y Q, Wang Z K, et al. Role of hunchback in segment patterning of Locusta migratoria manilensis revealed by parental RNAi. Dev Growth Differ, 2006, 48: 439-445

7 Patel N H, Hayward D C, Lall S, et al. Grasshopper hunchback expression reveals conserved and novel aspects of axis formation and segmentation. Development, 2001, 128: 3459-3472

8 Tautz D, Lehmann R, Schnürch H, et al. Finger protein of novel structure encoded by hunchback, a second member of the gap class of Drosophila segmentation genes. Nature, 1987, 327: 383-389

9 Simpson-Brose M, Treisman J, Desplan C. Synergy between the hunchback and bicoid morphogens is required for anterior patterning in Drosophila. Cell, 1994, 78: 855-865

10 Casares F, Sánchez-Herrero E. Regulation of the infraabdominal regions of the bithorax complex of Drosophila by gap genes. Development, 1995, 121: 1855-1866

11 Tautz D, Sommer R. Evolution of segmentation genes in insects. Trends Genet, 1995, 11: 23-27

12 Wu X, Vasisht V, Kosman D, et al. Thoracic patterning by the Drosophila gap gene, hunchback. Dev Biol, 2001, 237: 79-92

13 Clyde D E, Corado M S, Wu X, et al. A self-organizing system of repressor gradients establishes segmental complexity in Drosophila. Nature, 2003, 426: 849-853

14 Lehmann R, Nüsslein-Volhard C. hunchback, a gene required for segmentation of anterior and posterior regions of the Drosophila embryo. Dev Biol, 1987, 119: 402-417

15 Schröder R. The genes orthodenticle and hunchback substitute for bicoid in the beetle Tribolium. Nature, 2003, 422: 621-625

16 Pultz M A, Pitt J N, Alto N M. Extensive zygotic control of the anteroposterior axis in the wasp Nasonia vitripennis. Development, 1999, 126: 701-710

17 Pultz M A, Westendorf L, Gale S D, et al. A major role for zygotic hunchback in patterning the Nasonia embryo. Development, 2005, 132: 3705-3715

18 Mito T, Sarashina I, Zhang H, et al. Non-canonical functions of hunchback in segment patterning of the intermediate germ cricket Gryllus bimaculatus. Development, 2005, 132: 2069-2079

19 Dong Y, Friedric H M. Nymphal RNAi: systemic RNAi mediated gene knockdown in juvenile grasshopper. BMC Biotechnol, 2005, 5: 25

20 Ausubel F M, Brent R, Kingston R E, et al. Short protocols in molecular biology. 3rd ed. Beijing: Science Press, 1998. 414-422

21 Patel N H. Imaging neuronal subsets and other cell types in whole mount Drosohila embryos and larvae using antibody probes. In: Goldstein L S B, Fyrberg E A, eds. Methods in Cell Biology, Vol. 44. San Diego: Academic Press, 1994. 445-487

22 Chin C T, Chai C H, Sha C Y. Studies on the locust egg I: morphological changes of the embryo during egg incubation, with special reference to the developmental stages of the eggs collected from the field (Acridlldae: Orthoptera). Acta Entomol Sin, 1954, 4: 383-398

23 Isshiki T, Pearson B, Holbrook S, et al. Drosophila neuroblasts sequentially express transcription factors which specify the temporal identity of their neuronal progeny. Cell, 2001, 106: 511-521

24 Jurgens G, Wieschaus E, Nüsslein-Volhard C, et al. Mutations affecting the pattern of the larval cuticle in Drosophila melanogaster. Roux's Arch Dev Biol, 1984, 193: 283-295

Open Access This article is distributed under the terms of the Creative Commons Attribution License which permits any use, distribution, and reproduction in any medium, provided the original author(s) and source are credited. 